\title{
Radix nasi Transposition Flap for Medial Canthus and Nasal Sidewall Defects
}

\author{
Rodrigo Carvalho, MD, ${ }^{*}$ Diogo Casal, MD ${ }^{\dagger}$ Carlos Zagalo, MD, PhD $\stackrel{\ddagger}{\ddagger}$ and \\ José Rosa, MD, PHD
}

The authors have indicated no significant interest with commercial supporters.

$\mathrm{T}$ he head and neck are preferential locations for skin cancers, ${ }^{1}$ and when they involve different cosmetic units, they constitute a challenge for skin surgeons. An example of this is nasal sidewall and medial canthus area. Direct repair is possible for small defects, but for larger defects, local flaps or grafts are often required. In this anatomic area, the goals of reconstruction should be to obtain functional and aesthetic results, maintaining the normal concavity of the canthus with minimal distortion of the surrounding tissues. To obtain continuity of color and texture and reproduction of a natural appearance, reconstruction with a flap is frequently the first choice.

The classic banner-type transposition flap ${ }^{2}$ is a finger-shaped random-pattern cutaneous flap that makes use of areas of adjacent laxity. This flap allows for the placement of a long linear secondary scar in a skin fold or crease or along the junction of two cosmetic units. The authors decided to create a new application for this flap, using the skin reservoir that exists in the nose root (Radix nasi) for closure of medial canthus and nasal sidewall defects-the Radix nasi transposition flap. Upon retrospective analysis, this flap has been used successfully in six consecutive patients, with preservation of function and form. The technique and illustrative figures are included below.

\section{Technique}

The flap is harvested from the skin reservoir that exists in the nose root. The width of the flap is equal to the width of the defect and the length equal to the distance from the pivot point to the far edge of the defect. The flap is rotated in an arc about the pivot point between $60^{\circ}$ and $120^{\circ}$, according to the defect location. The secondary defect runs parallel and hidden in the natural skin folds existing between nose root and glabella. As the flap is rotated and transposed, nose convexity naturally corrects the eventually expected protrusion at the base of the flap. Any tissue redundancy that the rotating motion generates is carefully removed in a direction away from the pedicle of the flap such that the narrowing of the base of the flap does not compromise the blood flow. Even in cases in which larger length:width ratios are needed, vascular supply from supratrochlear artery branches is enough to maintain flap viability. The flap is sutured in place using a simple running suture with $5 / 0$ nylon that is removed 5 to 7 days

\footnotetext{
"Dermatology Department, Curry Cabral Hospital, Lisbon, Portugal; ${ }^{\dagger}$ Plastic and Reconstructive Surgery Department, São José Hospital, Lisbon, Portugal; *Plastic and Reconstructive Surgery Department, Portuguese Institute of Oncology, Lisbon, Portugal
} 


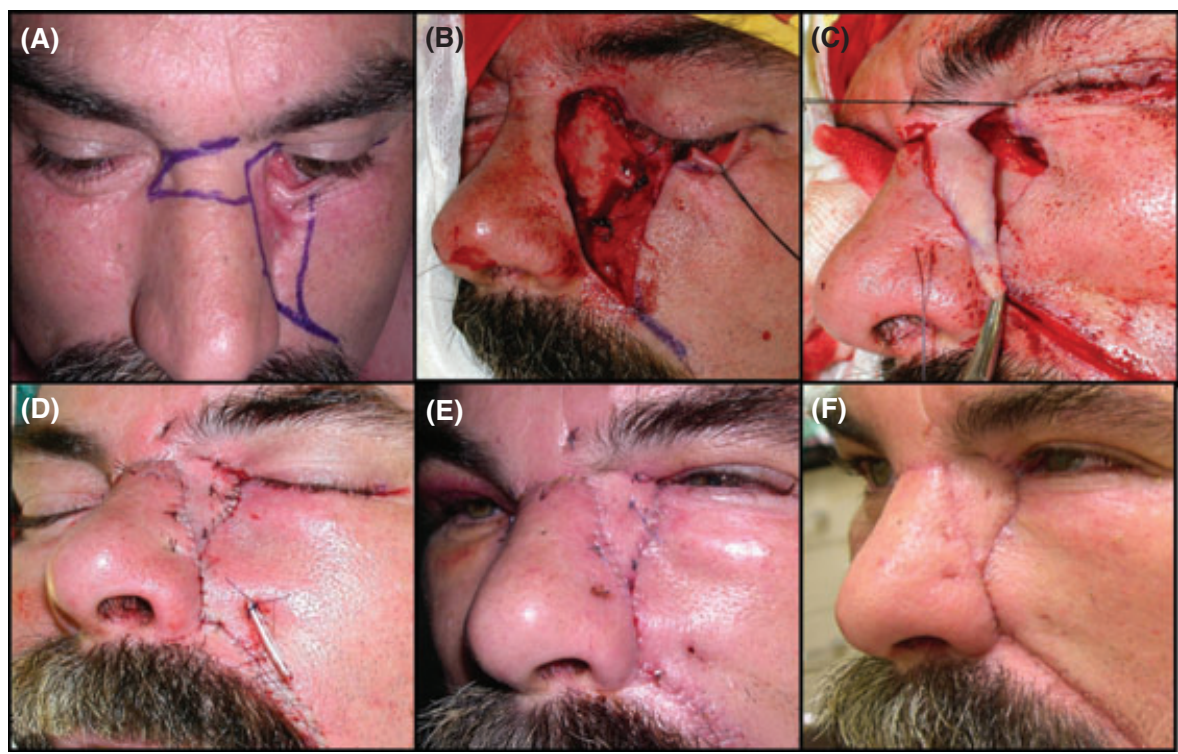

Figure 1. In patient 3, a large basal cell carcinoma was diagnosed at nasal sidewall and medial canthus. The flap width was equal to the width of the defect, and the length was equal to the distance from the pivot point to the far edge of the defect (A). Large defect created after tumor excision (B). The flap was rotated $90^{\circ}$ in an arc about the pivot point; in this case, with a large length:width ratio needed, vascular supply from supratrochlear artery branches was enough to maintain flap viability (C). The secondary defect was hidden in natural skin folds (D). At the time of suture removal, some edema is seen but no sign of flap necrosis (E). One week after suture removal, from a functional and cosmetic point of view, the result was very satisfactory $(F)$.

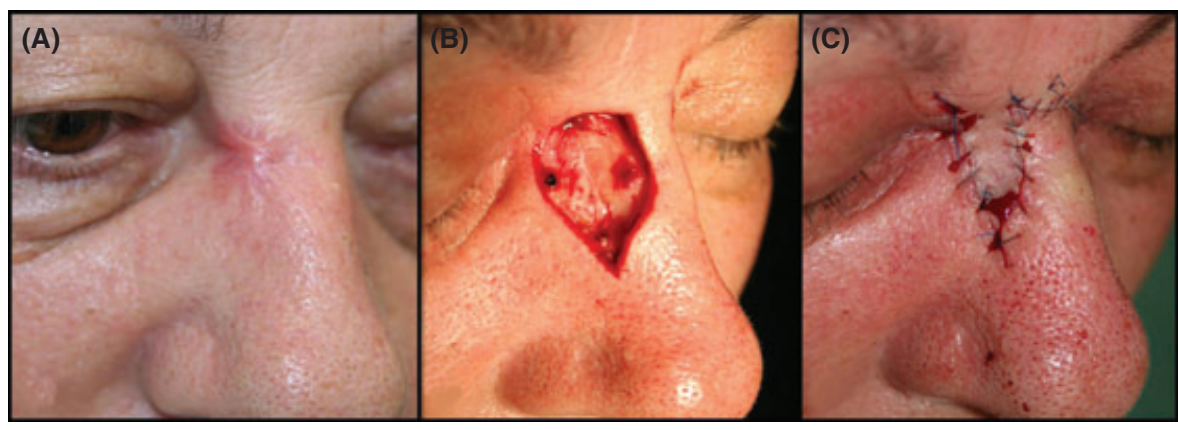

Figure 2. In patient 4, a basal cell carcinoma was diagnosed at nasal sidewall (A). After tumor excision, the defect in the upper half of nasal sidewall (B). Flap in place after $90^{\circ}$ rotation, with incision lines hidden in natural skin folds (C).

postoperatively. Because of the extensive size of the defect, an anchoring suture in the flap was placed in one patient (Figure 1).

The closure of the defect is achieved in a singlestage surgery. Medial canthus concavity was maintained, and the incisions used rapidly became unnoticeable and easily merged with natural wrinkles and folds (Figures 2, 3 and 4). Long-term follow-up has shown satisfactory to excellent cosmetic and functional results, without any complications.

\section{Patients}

Six patients with locally advanced malignant tumors of the nasal sidewall, medial canthus, or both who underwent surgical resection with curative intent were repaired using this technique (Table 1). 


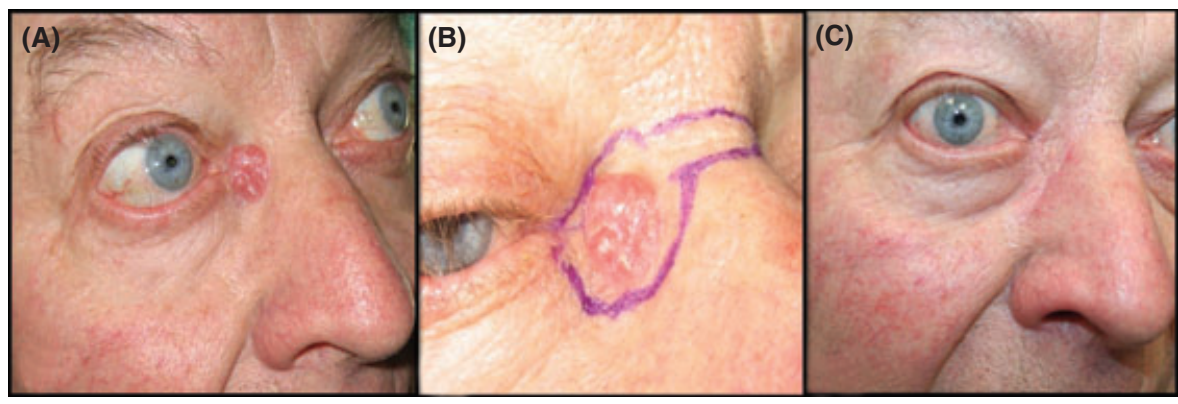

Figure 3. In patient 5, a basal cell carcinoma diagnosed at nasal sidewall and medial canthus (A). Flap design, with $120^{\circ}$ rotation necessary for defect closure (B). Follow-up 6 months after surgery with excellent functional and cosmetic result (C).

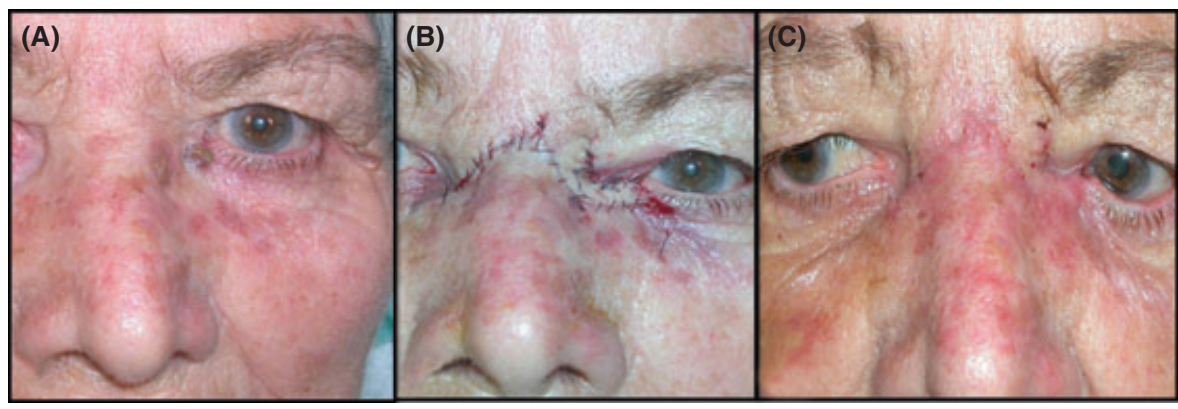

Figure 4. In patient 2, a basal cell carcinoma diagnosed at medial canthus (A). Flap in place after $120^{\circ}$ rotation, with incision lines hidden in natural skin folds (B). Follow-up weeks after surgery with excellent functional and cosmetic results (C).

\section{TABLE 1. Patient Details}

\begin{tabular}{lclllll} 
Patient & Age (years) & Sex & Pathology & Location & $\begin{array}{l}\text { Defect } \\
\text { Size (cm) }\end{array}$ & $\begin{array}{c}\text { Follow-up } \\
\text { (months) }\end{array}$ \\
\hline 1 & 72 & Male & BCC & NS+MC & $1.5 \times 1.5$ & 36 \\
2 & 80 & Female & BCC & MC & $1.5 \times 1.5$ & 28 \\
3 & 52 & Male & BCC & NS+MC & $4.0 \times 5.0$ & 32 \\
4 & 64 & Female & BCC & NS & $2.0 \times 3.0$ & 19 \\
5 & 66 & Male & BCC & NS+MC & $2.0 \times 2.0$ & 13 \\
6 & 63 & Male & BCC & NS+MC & $1.5 \times 1.5$ & 21 \\
\hline
\end{tabular}

BCC, basal cell carcinoma; NS, nasal sidewall; MC, medial canthus.

\section{Discussion}

The repairs of defects where different cosmetic units are involved constitute a challenge to skin surgeons. A variety of local flaps can be used to reconstruct defects of the medial canthus area, with various advantages and disadvantages, including the eyelid advancement, cheek rotation, rhomboid, bilobular, nasolabial, transposition glabellar, V-Y sliding or pedicle glabellar, and forehead flaps. ${ }^{3,4}$
Transposition flaps take advantage of regional laxity by mobilizing tissue from an adjacent area of excess laxity into the defect. They redistribute and redirect of tension and tend to be smaller than advancement and rotation flaps. Resultant scars are geometric broken lines that may be less conspicuous and tend to be easy to hide. Many different types of transposition flaps exist, with innumerable variations. The most commonly used 
transposition flaps include the classic rhombic flap, as described by Limberg, modified rhombic flaps, (Webster $30^{\circ}$ angle and the Dufourmentel flap), and banner-type flaps.

The Radix nasi transposition flap proposed herein combines the advantages and simplicity of the transposition banner flap, with the advantage of using the immediate adjacent skin of the nose root as donor. Like all transposition "banner" flaps is a fingershaped random pattern cutaneous flap that makes use of areas of adjacent laxity and allows for the placement of a long linear secondary scar in a skin fold or crease along the junction of two cosmetic units. This concept has already been used with good results in the classic nasolabial transposition flap, bilobed nasal flap and ear "banner" flaps.

In conclusion, the Radix nasi transposition flap for medial canthus and nasal sidewall defects presents several significant advantages. It allows reconstruction in a single-stage procedure. It achieves good aesthetic results because it uses ideally colored and textured skin for reconstruction. Moreover, the associated surgical scars become relatively inconspicuous because they are placed in natural wrinkles and folds.

\section{References}

1. Franceschi S, Levi F, Randimbison L, Lavecchia C. Site distribution of different types of skin cancer: new aetiological clues. Int J Cancer 1996;67:24-8.

2. Masson JK, Mendelson BC. The banner flap. Am J Surg 1977;134(3):419-23.

3. Mustardé JC. Tissue loss-deep. In: Mustardé JC editor. Repair and reconstruction in the orbital region (3rd ed). Edinburgh: Churchill Livingstone; 1991. pp. 76-89, 265-81, 549-53.

4. Patrinely JR, Marines HM, Anderson RL. Skin flaps in periorbital reconstruction. Surv Ophthalmol 1987;31:249-61.

5. Fortier-Riberdy G, Gloster HM. Reconstruction of the superior helical rim with a postauricular transposition flap. Dermatol Surg 2005;31:99-101.

Address correspondence and reprint requests to: Rodrigo Araújo Carvalho, MD, Serviço de Dermatologia, Hospital Curry Cabral, Rua da Beneficiência nº, 1069-639 Lisbon, Portugal, or e-mail: rodrigoaraujocarvalho@gmail.com 